\title{
EFECTOS AMBIENTALES DE DIFERENTES TIPOS DE CORTAS EN BOSQUE NATIVO. EL CASO DE LAS CORTAS DE PROTECCION EN
}

\author{
FAJAS
}

\author{
Luis Otero $D .(*)$ \\ Alvaro Contreras J. (**) \\ Luis Barrales M. (**)
}

\section{RESUMEN}

Esta investigación tiene por objetivo evaluar los efectos ambientales de las cortas de protección en fajas, siendo parte de una investigación más amplia sobre los efectos ambientales de los diferentes tipos de corta que se aplican al bosque nativo.

Las mediciones corresponden a variables microclimáticas tales como: luz difusa, temperatura del aire, temperatura del suelo, humedad relativa y capacidad evaporativa del aire. Las mediciones se realizaron en bosques del tipo forestal Coihue-Rauli-Tepa, en el predio Pilmaiquén en la Comuna de Panguipulli (Valdivia).

Las intervenciones realizadas consisten en la corta en fajas que se disponen en curvas de nivel y son de anchos variables, correspondientes a múltiplos de la altura del dosel dominante $(30,60$ y $90 \mathrm{~m})$. Las fajas cortadas se alternan con franjas de bosque de protección de $40 \mathrm{~m}$ de ancho. El estudio se basa en la comparación de un faja intervenida con el bosque intocado de la faja de protección. Para ello se utilizan funciones matemáticas que permiten evaluar y predecir el efecto de este tipo intervenciones.

Las conclusiones del estudio revelan que existe un efecto de protección lateral del bosque hacia la faja intervenida, que permite mantener en cierta medida las condiciones ambientales del bosque original. Estas condiciones ambientales varian de acuerdo a los diferentes anchos de las fajas. Las cortas equivalentes a 1 y 2 veces la altura del dosel (30 y $60 \mathrm{~m}$ en el caso estudiado), parecen ofrecer las mejores condiciones desde el punto de vista ambiental. 


\section{ABSTRACT}

The objective of this research is to evaluate the environment effects caused by strip protection cuts done as part of a larger study concerned with the environmental effects of the different cutting practices applied to native forests.

Measurement of microclimatic variables such as diffuse light, air temperature, soil temperature and air evaporative capacity were undertaken. The measurements were taken in forests of the type Coihue-Rauli-Tepa, at the Pilmaiquen farm located in the Panguipulli Comune (Valdivia)

The treatments consisted in strip cuttings which were oriented according to level curves and had variable width equivalent to multiple times the hight of the dominant crown $(30.60$ and $90 \mathrm{~m})$. The strips cut down alternate with strips of protection forests of $40 \mathrm{~m}$ width. The study consisted in comparing the treated strip with the untouched forest of the protection strip. To do this, mathematical functions were used that make it possible to evaluate and predict the effect of this kind of interventions

The study concludes that there is a lateral protective effect of the forest towards the strip being cut, that allows to mantain in a certain proportion the environmental conditions observed in the original forest. These environmental conditions vary according to the different width given to the strips. Cuts equivalent to 1 or 2 times the hight of the crown (30 to $60 \mathrm{~m}$ in the case studied), seem to offer the best conditions from an environmental point of view 


\section{INTRODUCCION}

Los diferentes tipos de cortas o métodos aplicables al bosque nativo definidos en la legislación forestal (Decreto Ley 701) han sido poco evaluados desde la perspectiva ambiental, en el sentido de saber qué efectos provocan estas intervenciones a nivel del microclima, suelo, flora y fauna del bosque.

En el pais los estudios ambientales del bosque normalmente están enfocados como estudios ecológicos, no vinculados a intervenciones silvicolas. Entre los estudios y publicaciones de carácter ambiental más relacionados a los problemas silvícolas, destacan los realizados por los siguientes autores: Donoso (1990); Huber et al (1984), Huber y Oyarzun (1989); Marchant (1984); Grosse (1988); Inostroza (1986); Espinoza (1972) y Amz, Delmastro y Schlatter (1969).

El sistema de cortas en fajas ha sido evaluado en sus aspectos silvicolas, en el tipo forestal siempreverde de la Cordillera de los Andes, por la Universidad Austral de Chile (Donoso, 1989). Los resultados obtenidos en este estudio muestran que la regeneración natural de especies colonizadoras y semitolerantes obtiene sus mejores resultados, en cuanto a crecimiento y densidad, bajo este sistema comparado con el método de selección, tala rasa y cortas de protección uniforme.

Experiencias realizadas en países como Perú y Costa Rica (Ocaña-Vidal, 1992; Dykstra y Heinrich, 1992), plantean que el método en fajas presenta importantes ventajas ecológicas, genera condiciones adecuadas para la regeneración del bosque y tiene efectos positivos de significación en los rendimientos y reducción de costos.

En relación a los costos de cosecha, estimaciones preliminares, realizadas por los autores, muestran que con tractor forestal la corta en fajas presenta costos de madereo un $35 \%$ menor que con el método de protección uniforme. Donoso (1989) también concluye que el método de cortas en fajas presenta menores costos operacionales que otros sistemas de cosecha, ya que presenta todas las ventajas de una tala rasa. 
Según Hawley y Smith (1972) otra ventaja silvicola que presenta este sistema es la generación de condiciones ambientales más diversas, de acuerdo a la distancia al borde del bosque protector. Esto permite el desarrollo de especies de distinto nivel de tolerancia a la sombra. Desde otro punto de vista. los daños a la regeneración son mínimos al momento de la cosecha del bosque de protección, ya que se pueden extraer los árboles a través de las fajas no taladas. Otros autores como Troup (1952), citado por Hawley y Smith, han demostrado las ventajas de las cortas en franjas para resolver diversos problemas silvícolas.

Las nuevas tendencias en la silvicultura, apoyadas sobre bases ecológicas, coinciden con las ventajas de las corta a tala rasa en pequeñas superficies, ya que permiten aumentar la diversidad del paisaje en el espacio y en el tiempo (Kimmins, 1993; citado por Bellefleur).

La combinación de áreas en etapas sucesionales diferentes. es decir de etapas tempranas representadas por el área cortada y etapas tardias representadas por el bosque adulto de la faja de protección, permite mantener e incluso incrementar la biodiversidad de las zonas intervenidas (Probst y Crow,1991)

Ecologos canadienses como Bellefleur (1994) recomiendan talas rasas en pequeñas superficies, en círculos o en fajas, ya que favorecen la regeneración de especies de sucesión secundaria y protegen adecuadamente el suelo.

El principal objetivo de este trabajo es establecer los efectos que provocan en el medio ambiente, específicamente en el microclima, las cortas finales bajo el método de Protección en Fajas o Cortas en Fajas.

Un estudio similar al que se presenta en este trabajo, fue desarrollado en un bosque sometido al método de Cortas de Protección Uniforme, con una cobertura residual del 30\% (Otero et al., 1994). En dicha investigación se concluye que los principales impactos se producen en los siguientes factores: luz difusa $(+441 \%)$, temperatura de suelo $(+236 \%)$, temperatura del aire $(+59 \%)$; humedad relativa del aire $(-37 \%)$ y capacidad evaporativa del aire $(+0,91 \%)$

Desde el punto de vista silvicola, los resultados también aportan antecedentes sobre las condiciones a las que tendrá que adaptarse fisiológicamente la regeneración natural o bien la plantación, luego de la corta. 
Los factores que determinan el crecimiento de los bosques y plantaciones presentan umbrales fisiológicos favorables o desfavorables para el desarrollo de diferentes especies.

Algunas investigaciones han revelado que ciertas coniferas tienen un crecimiento significativamente mayor cuando las temperaturas del suelo superan los $10^{\circ} \mathrm{C}$ y con temperaturas menores a $5^{\circ} \mathrm{C}$ las raices presentan resistencia al flujo del agua (Kaufmann, 1977). Según este mismo autor, en suelos con temperaturas de $15^{\circ} \mathrm{C}$, las raices y brotes presentan crecimientos óptimos en coníferas como el pino radiata.

En el caso del aire, temperaturas extremas de menos $2{ }^{\circ} \mathrm{C}$ durante dos noches seguidas provocan daños a la regeneración de ciertas coniferas, durante la primera temporada de crecimiento (Coates, Enmingham y Radosevich, 1991). Temperaturas optimas para la fotosíntesis han sido establecidas por Pollard y Logan (1977) para piceas, a nivel de $19{ }^{\circ} \mathrm{C}$ y con intensidades lumínicas de 22 mil lux. Según Weares y Clements (citado por Donoso, 1990) las temperaturas más favorables para especies de climas templados se encuentran entre 16 y $32{ }^{\circ} \mathrm{C}$. Desde el punto de vista de la germinación de las semillas el óptimo fluctúa entre 15 y $30^{\circ} \mathrm{C}$ (Baker, citado por Donoso, 1990).

Otros estudios que vinculan la sobrevivencia de plantas con valores de luminosidad, plantean que los Nothofagus, al igual que algunos Fagus, presentan alta sobrevivencia con luminosidades del orden de 40 a $50 \%$ de la existente a campo abierto (Grosse, 1988). En relación a este mismo factor, Ronco (1970) establece rangos de luminosidad de $\mathbf{4 0}$ mil a 50 mil lux como optimos para la fotosintesis de especies tolerantes (Picea) y sobre 100 mil lux. establece que se producen problemas de mortalidad en plantaciones. Donoso (1990) establece que el consumo de CO2 para la fotosintesis se incrementa hasta un límite óptimo de $\mathbf{3 0}$ a $\mathbf{5 0}$ mil lux para la mayoría de las plantas. 


\section{MATERIAL Y METODO}

\section{Enfoque Metodológico.}

El enfoque metodológico del proyecto está basado en la utilización de areas testigo y su comparación con las zonas intervenidas, mediante la instalación de estaciones de medición en transectos perpendiculares a las fajas, método utilizado comúnmente para este tipo de estudios (Nuñez y Sander, 1981; Marchant, 1984).

Con la información obtenida se construyen funciones matemáticas para cada factor analizado y para cada tipo de faja intervenida.

\section{Area de Estudio.}

El área de estudio se encuentra ubicada al NE de la ciudad de Panguipulli, Décima Región. Las coordenadas geográficas son: $72^{\circ} 50^{\prime} \mathrm{LS}$ y $40^{\circ} 00^{\circ} \mathrm{LW}$. La altitud es de 870 a $1050 \mathrm{msnm}$.

\section{Geomorfología y Topografia.}

El ensayo se estableció en una ladera de exposición norte. Las pendientes predominantes para cada una de las fajas son las siguientes: de $30 \%$ para la faja de $30 \mathrm{~m}, 38 \%$ para la faja de $60 \mathrm{~m}$ y $31 \%$ para la faja de $90 \mathrm{~m}$ de ancho. 
Clima.

La zona se caracteriza por su clima de montaña y presenta una elevada precipitación media anual, de aproximadamente $4400 \mathrm{~mm}$, con un mínimo de $180 \mathrm{~mm}$ en el mes de enero y de $669 \mathrm{~mm}$ en el mes de junio. Una parte de estas precipitaciones cae en forma de nieve, durante los meses de otoño e invierno.

La temperatura media anual es de $11,3^{\circ} \mathrm{C}$, en verano sube hasta $16^{\circ} \mathrm{C}$ y en invierno el promedio baja a $6,5^{\circ} \mathrm{C}$. La humedad relativa media anual es de $79 \%$, durante el verano baja al $76 \%$ y en invierno es de $85 \%$. En general las temperaturas medias máximas están comprendidas entre los 9 y $21^{\circ} \mathrm{C}$, dependiendo de la altura ${ }^{1}$.

Suelos.

Los suelos del área corresponden a Trumaos, desarrollados sobre arenas y escoria volcánica de diversa granulonetría. Estos suelos presentan alto contenido de materia orgánica en el horizonte superficial $(20 \%$ aproximadamente), una textura generalmente franco arenosa, un alto poder fijador de fosfatos, una alta permeabilidad e infiltración y una baja densidad aparente, menor de $0,8 \mathrm{~g} / \mathrm{cm} 3$ (Peralta y Oyanedel, 1980). 


\section{Vegetación.}

La zona en que se desarrolla el trabajo, corresponde a un bosque del tipo forestal Coihue-Rauli-Tepa de altura, en el cual la tepa a sido reemplazada por lenga, con una presencia mayoritaria de coihue. Se trata en términos generales de un bosque en estado maduro, con escasa regeneración y que ha sido sometido a floreo, particularmente del raulí.

Los árboles más viejos de estos bosques tienen edades que fluctúan entre 200 y 500 años, pero naturalmente se encuentra toda la gama de edades (Donoso, 1981).

En la Figura № 1 se puede ver el perfil vertical del bosque y su distribución horizontal. 


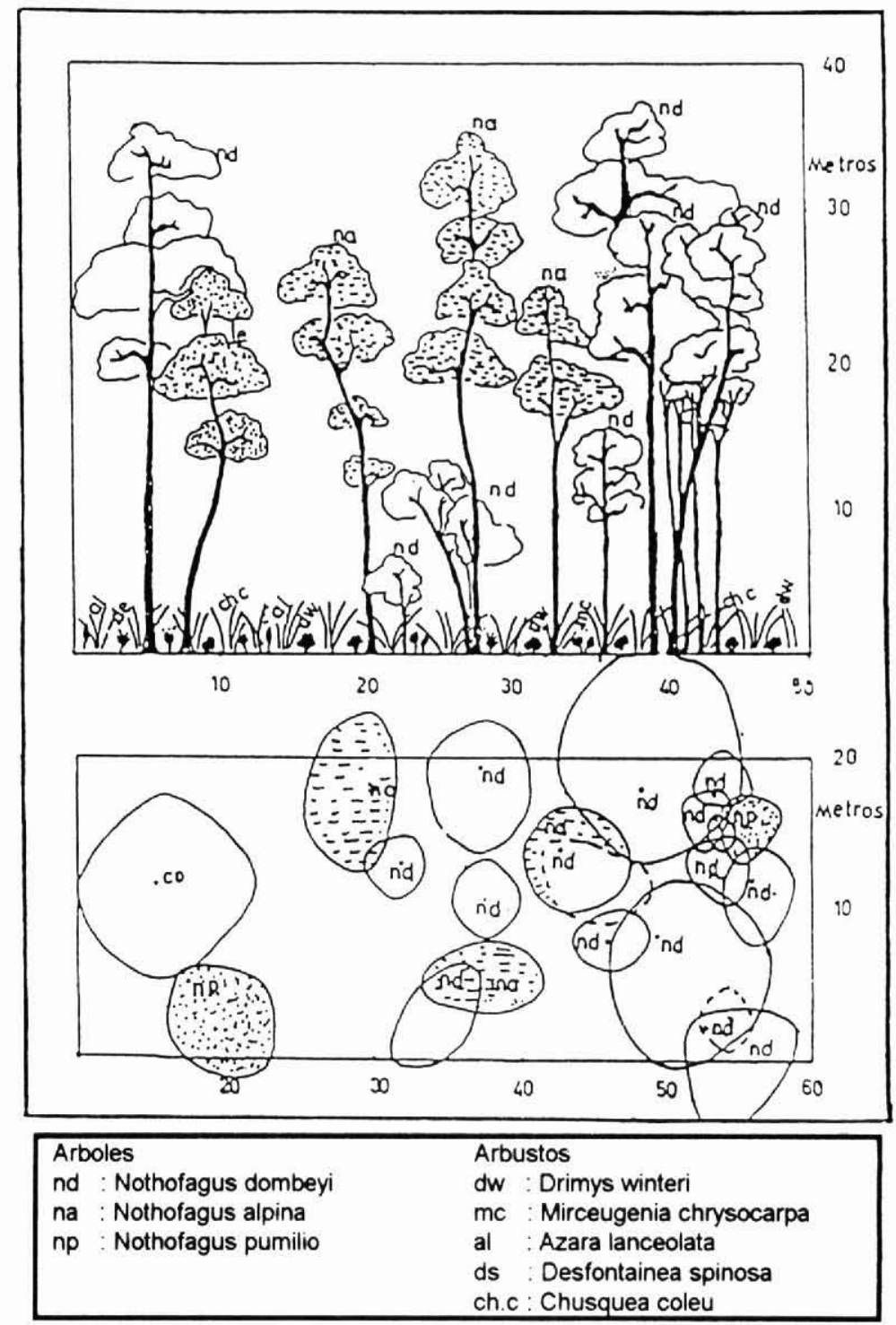

Figura $N^{\circ} 1$ PERFIL VERTICAL Y DISTRIBUCION HORIZONTAL BOSQUE COIHUE-RAULI-LENGA 
En el Cuadro $N^{0} 1$ se aprecian los parámetros del bosque intervenido.

\section{Cuadro $N^{0} 1$}

PARAMETROS DE RODAL DEL BOSQUE

\begin{tabular}{|l|c|c|c|c|c|}
\hline \multicolumn{1}{|c|}{ Especie } & \multicolumn{2}{|c|}{ DAP } & $\begin{array}{c}\text { Area Basal } \\
\left(\mathrm{M}^{2} / \mathrm{ha}\right)\end{array}$ & $\begin{array}{c}\text { Regeneración } \\
(\mathrm{N} / \mathrm{ha})\end{array}$ & $\begin{array}{c}\text { Cobertura } \\
(\%)\end{array}$ \\
\hline Coihue & 170 & 55,3 & 59,1 & 278 & - \\
Rauli & 30 & 58,0 & 8,0 & & - \\
Lenga & 10 & 56,0 & 2,5 & & - \\
\hline Total & 210 & & 69,6 & & 56,4 \\
\hline
\end{tabular}

DAP : promedio.

Periodo del Estudio.

La investigación se llevó a cabo entre enero y marzo de 1994, en días despejados sin nubosidad.

Diseño del Estudio.

Para la obtención de la información en terreno se establecieron en cada faja intervenida de 30,60 y 90 metros, estaciones de medición ambiental, en un transecto perpendicular a la faja. Las estaciones de medición se establecieron cada $15 \mathrm{~m}$ a partir del borde del bosque de protección, además de la estación establecida al interior de este bosque.

El número de estaciones de medición fue de 4 para la faja de $30 \mathrm{~m}, 6$ en la faja de $60 \mathrm{~m}$ y 8 para la faja de $90 \mathrm{~m}$, incluidas las estaciones testigos localizadas en el bosque de protección. 


\section{Método de Cortas de Protección en Fajas.}

El método de protección en fajas consiste en altemar franjas de bosque con franjas de corta, en las cuales se extrae la totalidad del dosel. Estas fajas se realizan en curvas de nivel y sus anchos corresponden a múltiplos de la altura promedio del dosel $(h)$, que en este caso fueron $1 \mathrm{~h}(30 \mathrm{~m}), 2 \mathrm{~h}(60 \mathrm{~m})$ y $3 \mathrm{~h}(90$ $\mathrm{m})$. El largo de las fajas es variable y depende de las condiciones topográficas, estando limitado por el bosque de protección que corresponde a las quebradas. En este estudio el largo de las fajas fue de aproximadamente $150 \mathrm{~m}$ para los tres anchos de faja (Figura $\mathrm{N}^{\circ} 2$ ).

El ancho de la faja de bosque de protección fue constante y correspondió a $40 \mathrm{~m}$, que es la altura máxima que alcanzan los árboles de mayor desarrollo. A orilla de los caminos se dejaron $20 \mathrm{~m}$ de bosque, con el objeto de proteger los taludes y conservar el paisaje del lugar.

El volumen de estos bosques fluctúa bastante. En los bosques estudiados el volumen medido en trozos fue de $672,5 \mathrm{~m}^{3} / \mathrm{ha}$, del cual aproximadamente un $27 \%$ corresponde a coinue aserrable, un $10 \%$ a madera debobinable de la misma especie, un $13 \%$ a rauli calidad aserrable y un $50 \%$ a madera pulpable. principalmente de coihue.

El objetivo silvicola de la intervención es regenerar un bosque nuevo dominado por rauli, por medio de plantación. Este bosque será complementado con regeneración natural de semillas provenientes de la faja de protección de especies como coihue, mañío, tepa y lenga, constituyéndose un bosque mixto. similar al bosque original. 




Ep : Efectos protección (radiación desde el bosque, evapotranspiración, sombra)

Cs : Calor del suelo

Ra : Radiación atmósferica

Rs : Radiación suelo

Et : Evapotranspiración 


\section{Variables Analizadas e Instrumentos de Medición.}

Las variables microclimáticas consideradas son las siguientes:

Luz. El indicador escogido es luz difusa, debido a que sus mediciones varian menos que las de luz directa. Para la medición de este parámetro se empleó un luxímetro, ubicado a $1,5 \mathrm{~m}$ del suelo y una sombrilla.

Temperaturas del Aire. Las temperaturas del momento, máximas y minimas, fueron medidas con un termómetro de temperaturas extremas. Los termómetros se protegieron del sol directo, en aquellas horas que este incidia sobre el instrumento.

Temperatura del Suelo. Estos valores se obtuvieron con termómetros de suelo enterrados a $10 \mathrm{~cm}$ de profundidad.

Capacidad Evaporativa del Aire. Esta se midió con evaporimetros de Piche, colocados a una altura de 1,5 $\mathrm{m}$ y protegidos del sol.

Humedad Relativa del Aire. Se registró con un higrómetro compacto portátil, equipado con termómetros de bulbo seco y húmedo.

Estas variables se analizaron en las tres fajas realizadas, con la excepción de la temperatura del suelo, la que solo se midió en fajas de $60 \mathrm{~m}$ y $90 \mathrm{~m}$.

Procesamiento y Análisis de la Información.

Los datos se procesaron mediante el programa Statgraphics versión 4.1, con el cual se realizaron regresiones lineales simples. Para cada regresión se obtuvieron sus parámetros y el coeficiente de regresión de Pearson y se graficaron las funciones, con sus respectivas bandas de confianza, a niveles de $p=0,05$ y $p=0,01$.

La pendiente de la recta dada por cada regresión, parámetro "b", se consideró como la intensidad del impacto y el parámetro "a" como el nivel del 
impacto. La pendiente representa los cambios o el diferencial entre el área intervenida y el bosque no alterado.

El parámetro "a", es el nivel en que se sitúa la recta y está relacionado con las condiciones ambientales estacionales, es decir con los promedios diarios de luminosidad, temperatura, humedad relativa, etc.

\section{RESULTADOS}

\section{Luz Difusa.}

La intervención a través de cortas en fajas genera, como es de esperar, un impacto fuerte en términos de mayores intensidades de luz difusa. Sin embargo debido al efecto de sombra del bosque de protección, el mayor impacto se observa en las fajas de 60 y $90 \mathrm{~m}$, siendo significativamente menor la cantidad de luz difusa en la faja de $30 \mathrm{~m}$ (Figuras $\mathrm{N}^{\circ} \mathrm{s} 3,4,5$ y 6). La pendiente de la regresión, o intensidad del impacto, para la faja de $30 \mathrm{~m}$ es de 1,56 y para las fajas 60 y 90 m fue de 3,62 y 3,9 , respectivamente (Cuadro $N^{\circ} 2$ ).

Los resultados indican que entre las fajas de mayor tamaño $(60$ y $90 \mathrm{~m})$ las diferencias en cuanto a la luz difusa son pocas y tienden a disminuir a mayor intensidad de luz. Con intensidades bajas las tres rectas tienden a juntarse, sin embargo mantienen niveles de luz mayor que el bosque original, el cual se oscurece antes por efectos de la cobertura.

El mayor nivel de luz existente en la faja de $60 \mathrm{~m}$ está dado por el parámetro "a" de la regresión, que presenta un nivel de 2.212 lux y es probable que se deba a las diferencias de pendiente, ya que esta faja presenta una pendiente mayor que la de $90 \mathrm{~m}$. Esta es la única regresión que presenta un situación de mayor efecto en una faja con respecto a otra de ancho mayor. 


\section{Cuadro $\mathrm{N}^{\circ} 2$}

\section{ESTADIGRAFOS DE LAS REGRESIONES}

\begin{tabular}{|l|c|c|c|c|c|}
\hline Variable & Faja & $a$ & $b$ & $r$ & $n$ \\
\hline Luz & $30 \mathrm{~m}$ & $1.170,6$ & 1,560 & 0,75 & 105 \\
\hline & $60 \mathrm{~m}$ & $2.212,0$ & 3,620 & 0,51 & 105 \\
\hline & $90 \mathrm{~m}$ & $1.452,0$ & 3,900 & 0,72 & 147 \\
\hline T. Aire & $30 \mathrm{~m}$ & 2,800 & 1,070 & 0,86 & 105 \\
\hline & $60 \mathrm{~m}$ & $-1,170$ & 1,260 & 0,58 & 75 \\
\hline & $90 \mathrm{~m}$ & $-5,670$ & 1,430 & 0,81 & 147 \\
\hline T. Suelo & $60 \mathrm{~m}$ & $-8,890$ & 2,100 & 0,62 & 96 \\
\hline & $90 \mathrm{~m}$ & $-5,840$ & 2,070 & 0,57 & 86 \\
\hline HR & $30 \mathrm{~m}$ & 0,311 & 0,954 & 0,94 & 84 \\
\hline & $60 \mathrm{~m}$ & $-3,970$ & 1,000 & 0,90 & 105 \\
\hline & $90 \mathrm{~m}$ & $-9,850$ & 1,056 & 0,86 & 147 \\
\hline CEA & $30 \mathrm{~m}$ & 1,068 & 0,838 & 0,61 & 90 \\
\hline & $60 \mathrm{~m}$ & 0,840 & 1,020 & 0,53 & 90 \\
\hline & $90 \mathrm{~m}$ & 0,470 & 1,477 & 0,56 & 126 \\
\hline
\end{tabular}
a: intercepto
b: pendiente
r. coeficiente de correlación
$n$ : número de datos 


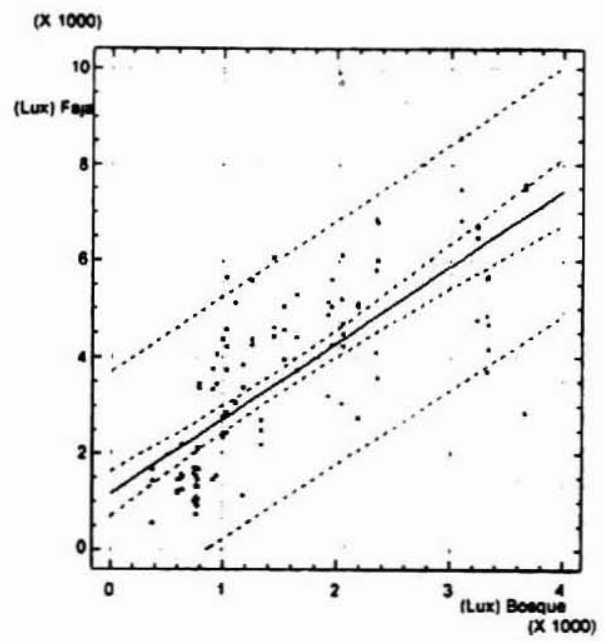

Figura N ${ }^{\circ}$ 3. EFECTOS DE LA CORTA EN LA FANA SOBRE LA LUZ DIFUSA. FANA DE 30 METROS

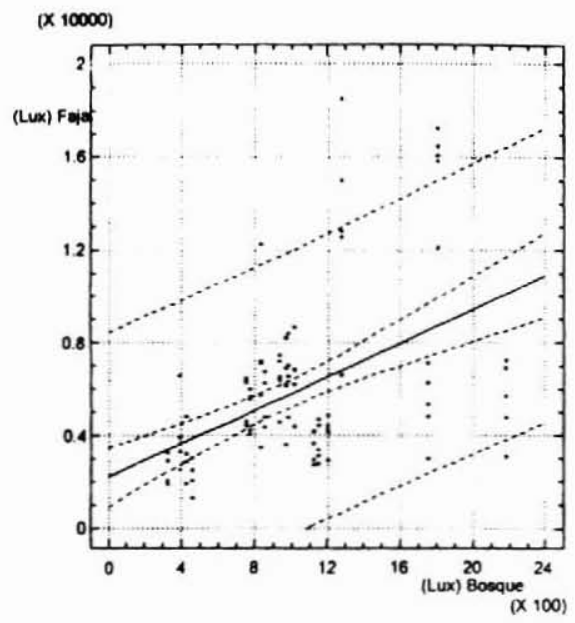

Figura $N^{\circ}$ 4. EFECTOS DE LA CORTA EN LA FANA SOBRE LA LUZ DIFUSA. FANA DE 60 METROS 


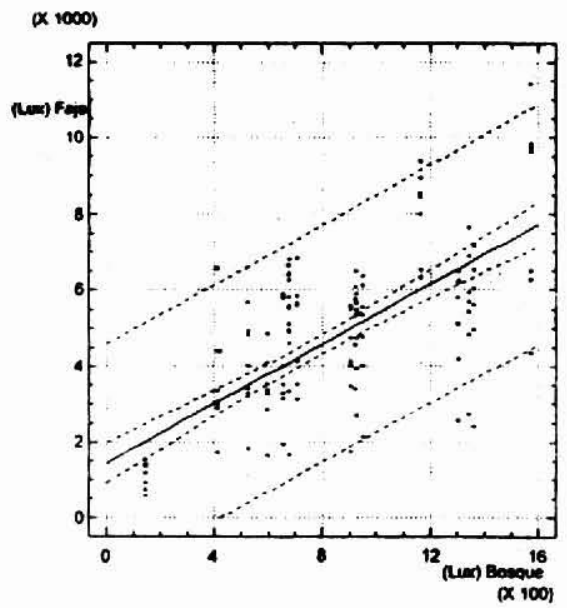

Figura N` 5. EFECTOS DE LA CORTA EN LA FAMA SOBRE LALUZ DIFUSA. FANA DE 90 METROS

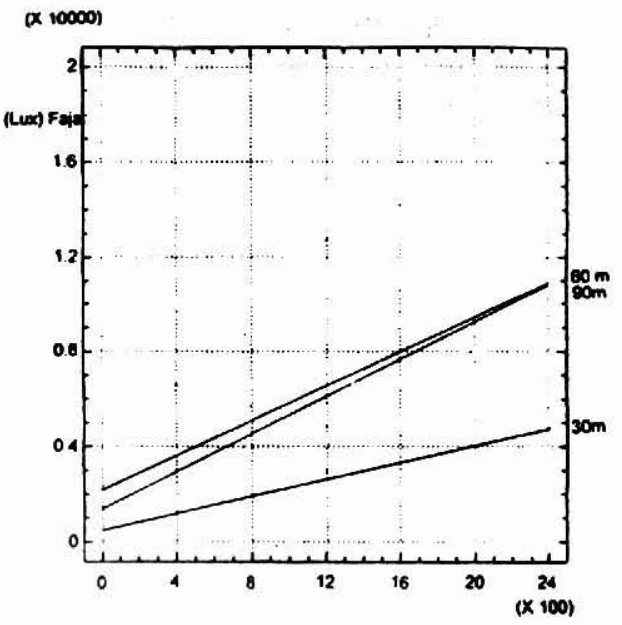

Figura $N^{\circ} 6$. COMPARACION DE REGRESIONES FAJA DE 30 METROS, 60 METROS Y 90 METROS 
Temperatura del Aire.

Como resultado directo de la mayor intensidad de luz provocada por la corta, se genera un aumento de las temperaturas del aire en las fajas, con respecto al bosque original. Este efecto tiende a disminuir a menor ancho de faja, debido al efecto de la sombra y de la evapotranspiración del bosque de protección.

De acuerdo a las pendientes de las curvas, la faja de $30 \mathrm{~m}$ presenta un impacto de una intensidad de 1,07, por lo que presenta condiciones bastante parecidas al bosque original. La faja de $60 \mathrm{~m}$ tiene un impacto de 1,26 y la de 90 m de 1,43 (Cuadro $N^{\circ} 2$ ).

De acuerdo a las funciones establecidas, a partir de los $24^{\circ} \mathrm{C}$ la faja de 60 m presenta temperaturas más altas que la de $30 \mathrm{~m}$. Por otra parte, la faja de 90 m muestra, de acuerdo a la regresión, mayores temperaturas que la de $60 \mathrm{~m}$ a partir de los $30^{\circ} \mathrm{C}$ (Figuras $\mathrm{N}^{\circ} 7,8,9$ y 10 ).

El valor negativo del intercepto "a" para las regresiones de 60 y $90 \mathrm{~m}$ refleja la mayor amplitud térmica o las temperaturas más extremas existente en estas fajas, ya que por ejemplo, para temperaturas inferiores a $14^{\circ} \mathrm{C}$ la faja de $90 \mathrm{~m}$ presenta valores menores que el bosque, lo mismo ocurre con la de $60 \mathrm{~m}$ cuando la temperatura del aire baja de $5^{\circ} \mathrm{C}$. 


\section{BIBLIOTECA INSTITUTO FORESTAL}



Figura $N^{0} 7$. EFECTOS DE LA CORTA SOBRE LA TEMPERATURA DEL ARE FAJA DE 30 METROS.

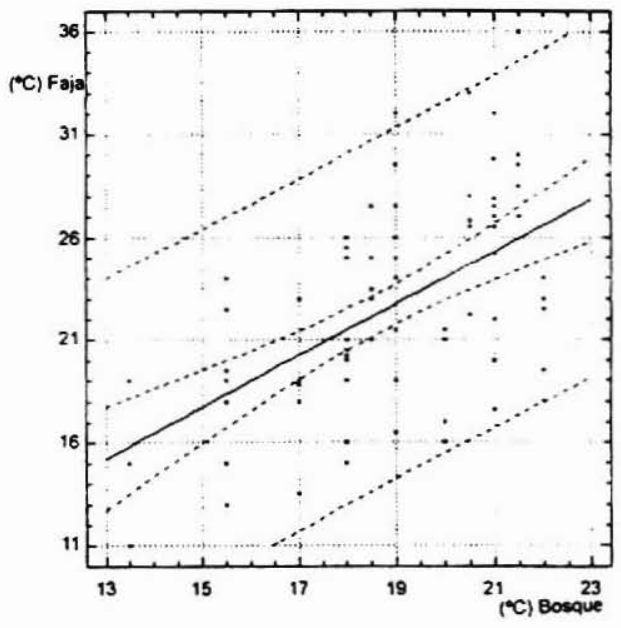

Figura $N^{\circ}$ 8. EFECTOS DE LA CORTA SOBRE LA TEMPERATURA DEL AIRE FAJA DE 60 METROS. 


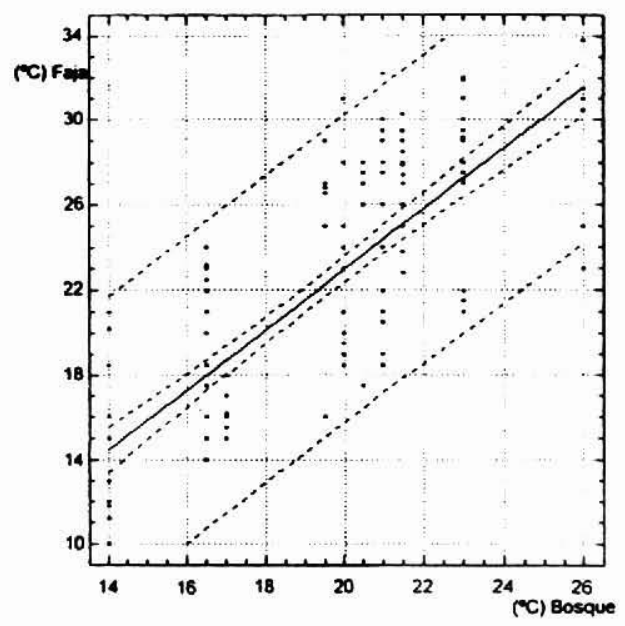

Figura № 9. EFECTOS DE LA CORTA SOBRE LA TEMPERATURA DEL ARE FANA DE 20 METROS.

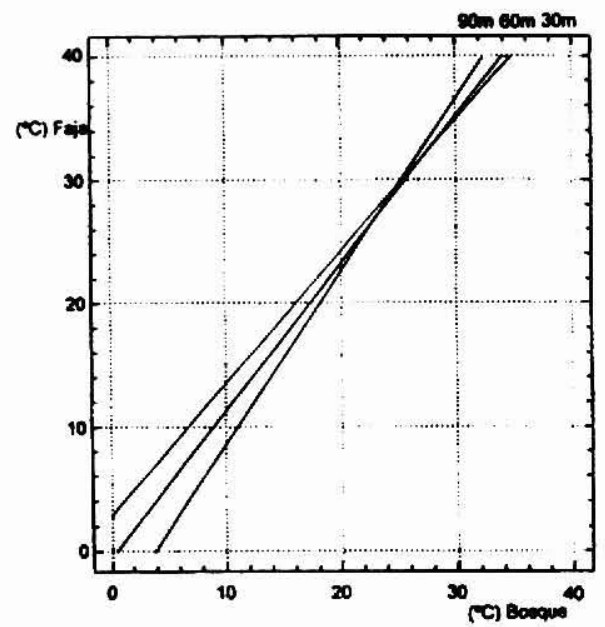

Figura $N^{\circ}$ 10. COMPARACION DE RECRESIONES FANA DE 30, 60 Y 90 METROS. 


\section{Temperatura del Suelo.}

La mayor radiación y temperatura del aire generada por la intervención, aumenta la temperatura del suelo en todas las fajas con respecto al bosque original.

Del análisis de las fajas de 60 y $90 \mathrm{~m}$, se concluye que el efecto de la corta con respecto a esta variable es el más intenso luego del cambio producido a nivel de la luz difusa. La pendiente o tasa de cambio de las temperaturas con respecto al bosque es de aproximadamente 2,1 para ambas fajas (Cuadro $\mathrm{N}^{\circ}$ 2). Sin embargo, las curvas se sitúan en niveles diferentes, la faja de 90 está aproximadamente 2,7 grados más arriba.

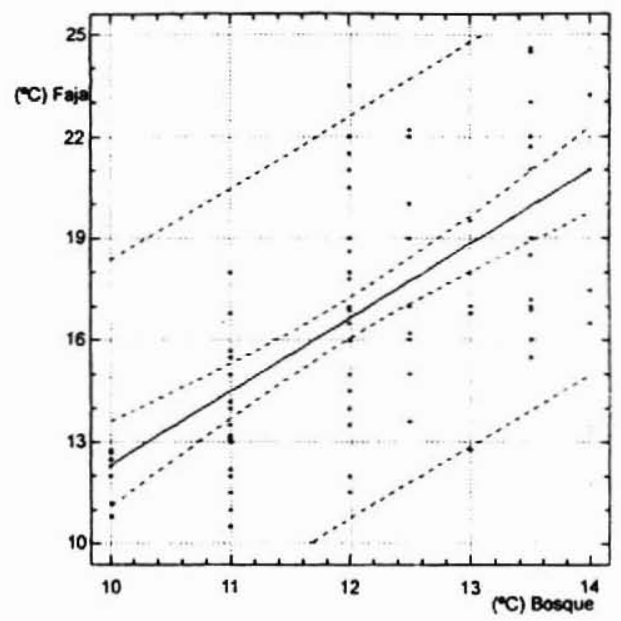

Figura No 11. EFECTOS DE LA CORTA SOBRE LA TEMPERATURA DEL SUELO FAJA DE 30 METROS. 


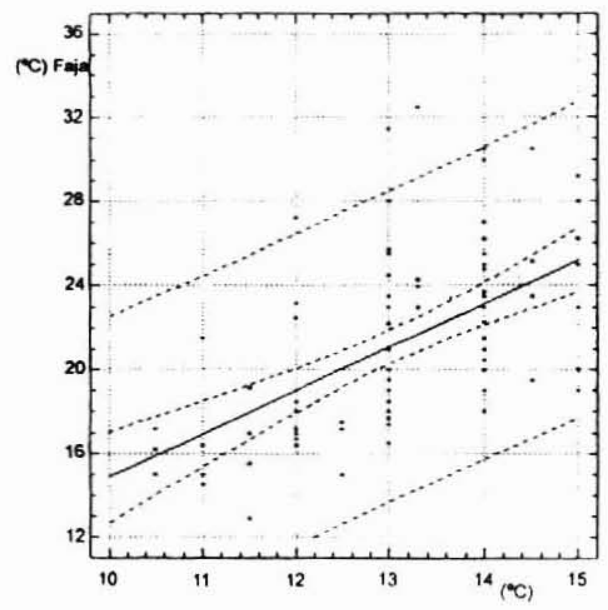

Figura $N^{\circ}$ 12. EFECTOS DE LA CORTA SOBRE LA TEMPERATURA DEL SUELO FANA DE $6 O$ METROS.



Figura No 13. COMPARACION DE REGRESIONES FAJA DE 60 METROS Y 90 METROS. 


\section{Humedad Relativa del Aire.}

El impacto de las fajas sobre la humedad relativa del aire, es relativamente bajo con respecto al bosque original, debido probablemente al efecto de la faja de protección, la cual mantiene un cierto nivel de humedad en el aire originado en la evapotraspiración del bosque.

Las curvas para cada una de las fajas analizadas muestran diferencias en sus pendientes. Para la faja de $30 \mathrm{~m}$ es de 0,954 y para las de 60 y $90 \mathrm{~m}$ es de 1 y 1,056, respectivamente, en consecuencia la intensidad del impacto es muy similar para los diferentes anchos de fajas (Cuadro $\mathrm{N}^{\circ} 2$ ).

Las diferencias entre las fajas de $30 \mathrm{~m}$ y $60 \mathrm{~m}$ tienden a anularse a mayor humedad relativa en los bosques, es asi como con humedades sobre el $72 \%$, prácticamente estas dos fajas presentan las mismas condiciones (Figuras $\mathrm{N}^{\circ}$ $14,15,16$ y 17$)$.

El valor negativo del parámetro "a" en las fajas de 60 y $90 \mathrm{~m}$ es teórico y solo representa las mayores diferencias existentes entre las fajas en condiciones de baja humedad relativa. 


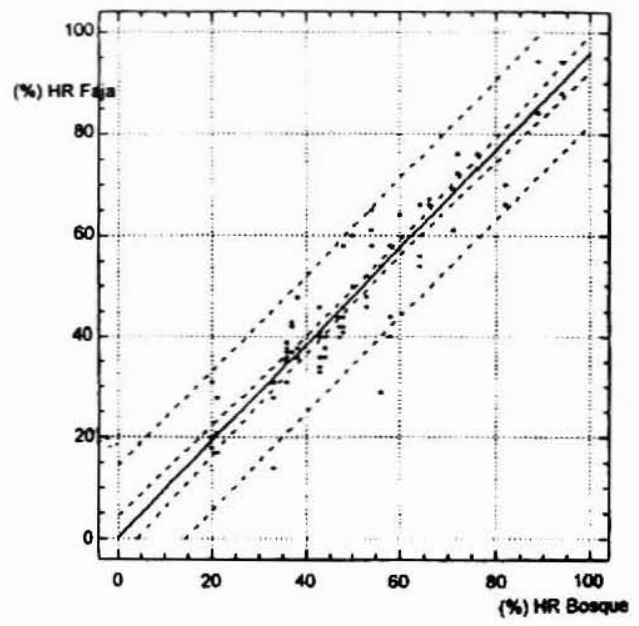

Figura $N^{\circ}$ 14. EFECTOS DE LA CORTA SOBRE LA HUMEDAD RELATNA DEL ARE FAJA DE 30 METROS.

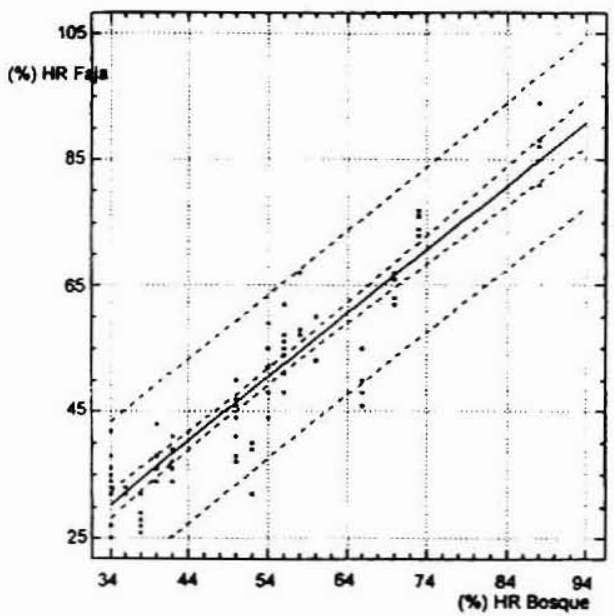

Figura № 15. EFECTOS DE LA CORTA SOBRE LA HUMEDAD RELATIVA DEL ARE FAJA DE 60 METROS. 


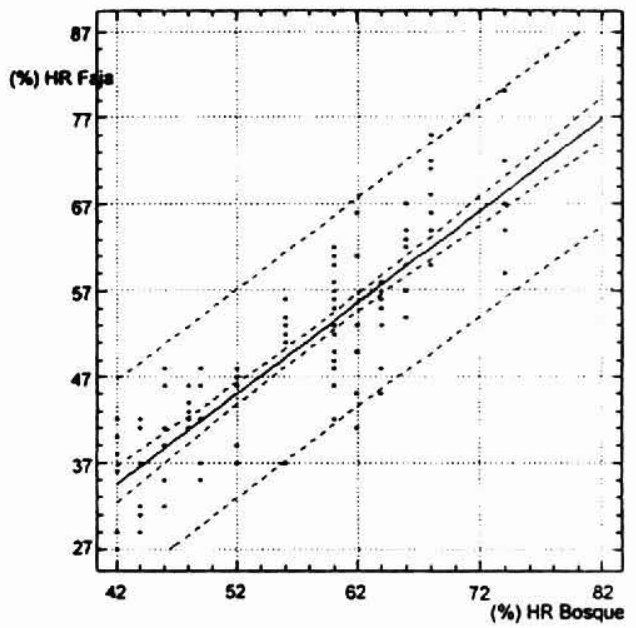

Figura $N^{\circ}$ 16. EFECTOS DE LA CORTA SOBRE LA HUMEDAD RELATNA DEL AIRE FANA DE 90 METROS



Figura $N^{\circ} 17$. COMPARACION DE REGRESIONES FAJA DE 30,60 Y 90 METROS. 


\section{Capacidad Evaporativa del Aire.}

El aumento de la temperatura en las fajas, así como la mayor circulación del aire, inciden en el incremento de la capacidad evaporativa del aire con respecto al bosque.

El impacto de las cortas sobre esta variable es relativamente bajo, las curvas presentan pendientes de 0,838 para la faja de $30 \mathrm{~m} ; 1,02$ para la faja de $60 \mathrm{~m}$ y 1,477 para la faja de $90 \mathrm{~m}$ (Cuadro $\mathrm{N}^{\circ} 2$ ).

En los Figuras $\mathrm{N}^{\circ} 18,19,20$ y 21 se muestra el impacto de la intervención en este factor. Se aprecia que las fajas de 30 y $60 \mathrm{~m}$ presentan entre si menor diferencia que con la faja de $90 \mathrm{~m}$.

La pendiente de 0,838 de la curva de la faja de $30 \mathrm{~m}$ indica que el impacto es menor cuando la capacidad evaporativa sube y las diferencias entre el bosque y el área cosechada tienden a anularse. El valor del parámetro "a" refleja el nivel inicial del impacto $(1,068)$.

Bajo niveles de evaporación de $0,8 \mathrm{ml}$ las relaciones entre las fajas se invierten y la mayor evaporación la presentan las fajas de menor ancho, esto se debe probablemente al aumento de la humedad relativa del aire en las tardes y en las mañanas, humedad que afecta a las fajas más anchas. 


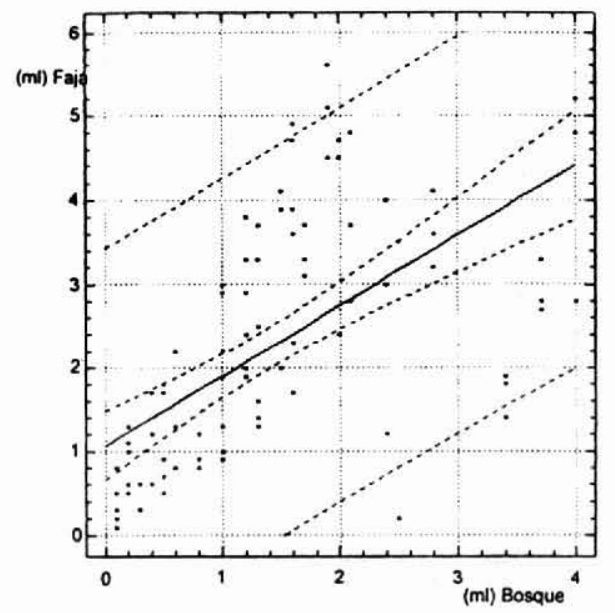

Figura № 18. EFECTOS DE LA CORTA SOBRE LA CAPACIDAD EVAPORATIVA DEL AIRE FAJA DE 30 METROS.



Figura № 19. EFECTOS DE LA CORTA SOBRE LA CAPACIDAD EVAPORATINA DEL ANRE FAJA DE 60 METROS. 




Figura $N^{\circ} 20$. EFECTOS DE LA CORTA SOBRE LA CAPACIDAD EVAPORATNA DEL AIRE FANA DE 90 METROS.

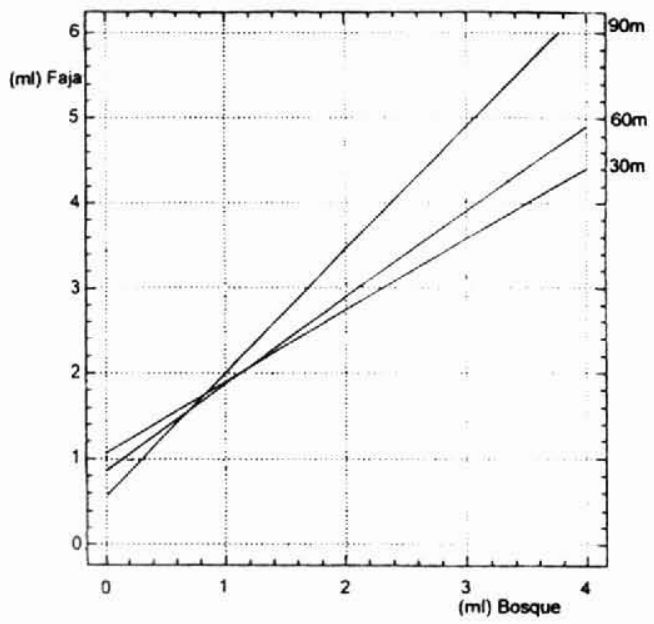

Figura $N^{0} 21$. COMPARACION DE REGRESIONES FAJA DE 30 METROS, 60 METROS Y 90 METROS. 


\section{DISCUSION}

El método de cortas analizado genera un efecto de protección lateral del bosque hacia la faja intervenida, que permite mantener en cierta medida las condiciones ambientales del bosque original. Estas condiciones ambientales varían de acuerdo a los diferentes anchos de las fajas.

Al aumentar el ancho de la faja de corta los factores que cambian con mayor intensidad son la luz y temperatura del suelo y, en menor medida, la temperatura del aire, capacidad evaporativa y humedad relativa del aire.

Los cambios producidos con respecto a la luz difusa en la faja de $30 \mathrm{~m}$, corresponden a 1,6 veces los existentes en el bosque y son bastante menores que los experimentados por las fajas de 60 y $90 \mathrm{~m}$, cuyas variaciones son de 3,6 y 3,9 veces, respectivamente.

En relación a las temperaturas del suelo las fajas de 60 y $90 \mathrm{~m}$ presentan intensidades de impacto similares, en ambas intervenciones el incremento de las temperaturas equivale a 2,1 veces la existente en el bosque. Sin embargo, los niveles de temperatura de la faja de $90 \mathrm{~m}$ son 3 grados superiores que aquellas de la de $60 \mathrm{~m}$.

Los impactos en las temperaturas del aire revelan cambios más significativos entre las intervenciones de 30 y $60 \mathrm{~m}$, que entre esta última y la faja de $90 \mathrm{~m}$. En la de $30 \mathrm{~m}$ la temperatura del aire incrementa 1,1 veces la del bosque, en la faja de $60 \mathrm{~m}$ aumenta 1,3 veces y en la de $90 \mathrm{~m} \mathrm{1,4}$ veces.

La intensidad del impacto en la capacidad evaporativa del aire es bajo para las fajas de 30 y $60 \mathrm{~m}$, las que varian en 0,83 y 1,02, respectivamente, en relación al bosque. Para la faja de $90 \mathrm{~m}$ la intensidad del impacto es de 1,5 veces la existente en el bosque.

La humedad relativa es el factor menos alterado con la intervención, sin embargo, para humedades relativas bajas, las fajas tienden a diferenciarse disminuyendo la humedad en forma más marcada en las fajas de 90 y $60 \mathrm{~m}$. La intensidad del impacto en la faja de $30 \mathrm{~m}$ corresponde a 0,95 veces el del bosque, para las fajas de 60 y $90 \mathrm{~m}$ la variación es 1 y 1,05, respectivamente, 
por lo que prácticamente no cambian. Por lo tanto el impacto está dado por el nivel inicial de $-9,8 \%$ de humedad relativa para la faja de $90 \mathrm{~m}$, de $-3,9 \%$ para la de $60 \mathrm{~m}$ y de $0,3 \%$ para la $30 \mathrm{~m}$.

Las regresiones lineales descritas son muy significativas y permiten predecir las variaciones microambientales que se producirán con la intervención silvicola durante el periodo de verano.

Los menores coeficientes de correlación encontrados fueron los correspondientes a la temperatura de suelos y a la capacidad evaporativa del aire. Por otra parte, en la faja de $60 \mathrm{~m}$ se presentan en general menores niveles de correlación en sus variables.

Dados los efectos observados, serían recomendables anchos de corta equivalentes a $30 \mathrm{~m}$ o $60 \mathrm{~m}$ ( 1 y 2 veces la altura del dosel) para los bosques y condiciones analizadas.

De acuerdo a los resultados obtenidos, las cortas en fajas con anchos equivalentes a 3 veces la altura del dosel $(90 \mathrm{~m})$, equivalen en términos de la intensidad del impacto (Otero et al,1994), a una corta de protección uniforme con una cobertura del $30 \%$.

\section{RECONOCIMIENTOS}

Para esta investigación se ha contado con la valiosa colaboración de la empresa BOMASA S.A. de Panguipulli, que ha dado las facilidades para la realización de las cortas en bosques de su propiedad, mostrando un permanente interés por la innovación y búsqueda de alternativas de manejo para el bosque nativo. 


\section{REFERENCIAS BIBLIOGRAFICAS}

Arnz J., Delmastro R. y Schlatter, J., 1969. Influencia de un Rodal de Pinus Radiata sobre Algunos Factores Ecológicos en Comparación al Campo Abierto. Tesis. UACH. Valdivia.

Bellefeur, Pierre., 1994. Las Bases Ecológicas del Desarrollo Forestal Sustentable. Seminario Medio Ambiente, Biodiversidad y Actividades Productivas. Instituto Forestal. Santiago 14-15 de Junio.

Coates K., Emmingham and Radosevich., 1991. Coniffer-seedlind Success and Microclimate at Different Levels of Herb and Shrubcover a Rhododendron-VacciniumMenziesia Community of South Central British Columbia. Can. Journal Forestry 21: 858866 .

Donoso, C., 1981. Tipos Forestales de los Bosques Nativos de Chile. Publicación FAO. Chile.

Donoso, C., 1989. Regeneración y Crecimiento en el Tipo Forestal Siempreverde Costero y Andino Tras Distintos Tratamiento. Silviculturales. Rev. Bosque Vol 10 No1 y No2. Valdivia.

Donoso, C., 1990. Ecologia Forestal. El Bosque y su Medio Ambiente. Facultad de Ciencias Forestales. UACH. Editorial Universitaria. Santiago.

Dykstra, D. y Heinrich, R., 1992. Sostenimiento de los Bosque Tropicales Mediante Sistemas de Explotación Ecológicamente Adecuados. Unasylva 169. Vol 43.

Espinosa, M., 1972. Efecto Importante Sobre la Regeneración del Rauli. Tesis Facultad Ciencias Forestales. UACH. Valdivia.

Grosse, A., 1988. Desarrollo de Rauli en Vivero Bajo Distintos Niveles de Luminosidad y Espaciamiento. Ciencia e Investigación Forestal. INFOR.

Hawley, R.C., Smith, D.M., 1972. Silvicultura Práctica. Ediciones Omega, S.A. Barcelona. 
Huber, A., Schlatter J. y Oyarzun C., 1984 Aporte en Elementos Nutritivos por la Hojarasca de Bosque Adulto de Pinus Radiata. Bosque 7(2)59-64.

Huber, A. Y Oyarzun C., 1989. Producción de Hojarasca y sus Relaciones con Factores Meteorológicos en un Bosque de Pinus radiata. Rev. Bosque (5)1 : 1-11.

Inostroza F., 1986. Estudio Micrometeorológico en un Bosque de Pino Radiata. Tesis. Facultad Ciencias Forestales. UACH. Valdivia.

Kaufmann, M. R., 1977. Soil Temperature and Drought Effects on Growth of Monterrey Pine. For. Sci. 23:317-324.

Kittrege, Joseph., 1973. Forest Influences. Dover Publication, Inc. New York. $394 \mathrm{Pg}$

Marchant, H., 1984. Estudio Micrometereológico en un Bosque Siempre Verde Intervenido con Distintos Métodos de Repoblación. Tesis. Facultad Ciencias Forestales. $\mathrm{UACH}$ Valdivia.

Nuñez. M, Sander. D., 1981. Frost Protection in a High Altitude Shelterwood. Department of Geography. University of Tasmania.

Ocaña-Vidal, J., 1992. Ordenación de Bosques Naturales Mediante Franjas Orotectoras. Unasylva 169, Vol 43

Otero, L. Donoso, P. y Barrales, L., 1994. Efectos Ambientales de las Cortas de Protección en Bosque Nativo. Seminario Medio Ambiente, Biodiversidad y Actividades Productivas. Instituto Forestal. Santiago 14-15 de Junio de 1994.

Pollard, D. F. W. and Logan, K. T., 1977. The Effects of Light

Intensity, Photoperiod, Soil Moisture Potencial, and Tempetarure on Bud Morphogenesis in Picea Species. Can. J.For. Res. 7:415-421.

Ronco, F., 1970 Influence of Ligth Intensity on Survival of

Planted Engelman Spruce. For. Sci. 16:331-339

Peralta, M. y Oyanedel, E., 1980. Regeneración en Bosque Nativo de

Raull. Facultad Ciencias Forestales. Universidad de Chile. Santiago.

Probst, J. and Crow, T., 1991. Integrating Biological Diversity and Resource Management. Journal of Forestry $89(2)$. 